Probably on of the most telling arguments in favour of both books is that one realizes that ultra-microanalysis, whether in the inorganic or the organic field, has no longer that noli me tangere connotation that it used to have. Like microanalysis thirty years ago, it now has the right to be included in the equipment of any general analytical laboratory.

I cannot refrain from closing these reviews with an almost irrelevant comment. The attribution of "firsts", profitless though it may be, is intriguing to anyone with a historical bent, but must be handled with the greatest caution. Prof. Korenman, in saying that "as early as 1868 , K. A. Timiryazev described some procedures for gas ultramicroanalysis", must surely be unaware that in 1855 Thomas Andrews described to the British Association the detection and measurement of hydrogen and oxygen from the electrolysis of water in amounts of approximately $10^{-4} \mathrm{~cm}^{3}$.

Cecil L. Wilson

\section{The Origin and Evolution of the Universe}

By Evry Schatzman. Translated from the French by Bernard and Annabel Pagel. Pp. $288+4$ plates. (London: Hutehinson and Co. (Publishers), Ltd., 1966.) 84s. net.

THIs is a translation of a book which first appeared in French in 1955. Some of the developments in astronomy and cosmology since then have been mentioned in the English version, but the rapid advances and discoveries of the past few years (such as quasars) have not been included. There is a fairly extensive account of the state of knowledge of stellar astronomy, and there are sections dealing with theories of the origin and evolution of stars, stellar systems and the solar system. The title of the book is perhaps a little misleading; cosmology as such occupies a relatively small proportion of the contents.

The overall planning of the book and the choice of subject-matter are excellent. In general, the treatment of the individual topics is quite good; the discussion, which is mainly non-mathematical, is readable. Unfortunately the book contains a rather large number of careless errors. Names are mis-spelt and references are incorrect. There are also some inconsistencies of a more serious nature. To name one, on pages 36 and 37 , shortperiod variables are assigned to the spherical component of the Galaxy without further comment; however, on pages 46 and 47 it is stated that the disk population includes $R R$ Lyrae variables. In reality, only some short-period variables are of the $R R$ Lyrae type; $R R$ Lyrae variables are probably represented in both the disk and in the spherical components. J. B. Alexander

\section{Plasma Physics}

(Lectures presented at the Seminar on Plasma Physics organized by and held at the International Centre for Theoretical Physics, Trieste, from 5-31 Oct., 1964.) Pp. 649. (Vienna: International Atomic Energy Agency; London: H.M.S.O., 1965.) 273 schillings; 78s.; \$13.

Approximately half of Plasma Physics is devoted to a series of introductory lectures. In these the important aspects of plasma physics are discussed by various authors both from the macroscopic points of view using the magnetohydrodynamic equations, and from the microscopic one. The latter includes a discussion of the selfconsistent Vlasov approximation and of the transport corrections. These lectures are individually of a high standard and give a comprehensive introduction to plasma physics but inevitably lack continuity.

The other half of the book contains lectures given by experts discussing work in which they are actively engaged. Although these lectures were given nearly 2 years ago they contain much material which is still relevant.
One topic of current interest is the theory and experimental work on the stabilization of plasma to lowfrequency (small compared to the ion cyclotron frequency) instabilities. A detailed discussion is presented of the stabilizing effects of Joffe bars, the concept of minimum $B$ and its generalization to toroidal systems.

Another topic which receives considerable attention is the problem of plasma turbulence. In the limit of weak turbulence the basis of quasi-linear theory and its extension to include mode-mode coupling terms is described and the application of these theories to the problems of enhanced diffusion and the structure of collisionless shocks discussed.

Two lectures, one on fully developed turbulence and the other on the kinetic theory of weakly unstable systems, give a clear indication of the more fundamental aspects of plasma physies.

These lectures, particularly those in the second half of the book, provide a very stimulating exposition of some of the present-day problems of plasma physies. The printing is of a high quality which makes the book easy to read though, of course, not necessarily easy to understand. This collection of lectures is to be thoroughly recommended both as an introduction to plasma physics and as a reference book to the present-day problems in this field.

G. Rowlands

\section{Testing of Polymers}

Vol. 1. Edited by John V. Schmitz. Pp. xiv +479. (New York and London: Interscience Publishers, a Division of John Wiley and Sons, 1965.) 147s.

In materials science and technology, testing is an essential requirement: it provides an experimental description of the material as a means for the objective appraisal and comparative evaluation of its performance. As a result of the diversity and explosive growth of synthetic polymers and the limitless chemical possibilities of providing tailor-made polymeric products of desirable properties, a unification and co-ordination of the wide variety of testing procedures has become desirable. In this series of publications an attempt has been made to provide an authoritative discussion of all aspects of testing and in a form suitable for use by polymer chemists and physicists, material scientists, engineers and manufacturers, all of whom need to understand and apply test data.

Volume $I$ of the series consists of fourteen chapters each of which covers some significant aspect of the testing of synthetic polymers. Most chapters review methods for evaluating individual properties, but other articles present background information or methods of evaluating individual properties, as well as service tests for end use purposes. The opening chapter discusses the purpose and the importance of standards, sources of standards, specification of materials and classification. This is followed by an account of the conditioning equipment for polymer testing. Chapters 3 and 4 which deal with the mechanical relationships in testing for the mechanical properties of polymers are concerned only with the basis of the tests and do not describe types and procedures for mechanical tests. The next five chapters (Chapters 5-9) cover in detail the wide subject of electrical property test methods and include d.c. dielectric conductance and conductivity, dielectric constant and loss measurements, electrical resistivity techniques and high voltage testing. The remaining chapters deal with specialized topics, testing for odour and taste, indentation and compression tests for floor coverings, cavitation erosion, and testing of gas and vapour permeation. The final chapter provides a useful literature coverage of selected references on sources of standards and tests for polymers.

This first volume is certainly a most valuable reference book and will be widely used by all interested in polymeric materials and their applications.

C. E. H. BAwN 